\title{
EVALUACIÓN DE VARIEDADES DE ARROZ EN LA ZONA ORIENTAL DE CUBA ${ }^{1}$
}

\author{
José Avila², Luis Manuel González ${ }^{3}$, Tania Obiol ${ }^{2}$
}

\begin{abstract}
RESUMEN
Evaluación de variedades de arroz en la zona oriental de Cuba. Se estudiaron 33 variedades de arroz: 15 de ciclo medio y 18 de ciclo corto sobre la base de su comportamiento agronómico, industrial y de resistencia a plagas y enfermedades. Las variedades de ciclo corto: IACuba-18 e IACuba-20, resultaron ser similares en rendimiento agrícola y resistencia a las principales plagas y enfermedades a la variedad Perla, usada como testigo, pero superiores en granos enteros y cristalinos, así como en el porcentaje de proteínas en el grano. Entre las variedades de ciclo medio se destacaron las variedades IACuba-17 e IACuba-19, que aunque no mostraron diferencias significativas en el rendimiento agrícola con la variedad testigo J-104, sí la superaron en el rendimiento industrial y la resistencia a enfermedades.
\end{abstract}

\begin{abstract}
Título en inglés. At Rice Research Station "Jucarito", with the purpose to select and to recommend the most appropriate for the easthern region of Cuba, 33 rice varieties: 15 of half cycle and 18 of short cycle, based of their agronomic, industrial behavior and resistance to pest and diseases, were studied. The results indicated that, the varieties of short cycle IACuba-18 and IACuba-20, turned out to be similar in agricultural yield and resistance to the main pest and diseases to the Perla, variety used as the control, but superiors in whole and crystalline grains, as well as in the grain proteins percentage. Among the varieties of half cycle they stood out the varieties IACuba-17 and IACuba-19 that although they didn't show significant differences in the agricultural yield with the control variety J-104, they overcame it in the industrial yield and the diseases resistance.
\end{abstract}

\section{INTRODUCCIÓN}

A lo largo de la historia, el mejoramiento de las plantas cultivadas ha ido alcanzando una mayor importancia, en el afan del hombre por lograr aumentos en la producción agrícola, que satisfagan las demandas cada vez más creciente de la sociedad (Diaz, Pérez y Morejon 1998). En tal sentido una importancia primordial se le atribuye a la selección de aquellos genotipos más satisfactorios, ya que el objetivo final de cada programa de mejora con fines comerciales, es la obtención de variedades que posibiliten un incremento productivo, ya sea desde el punto de vista cuantitativo o cualitativo.
El arroz constituye la base nutricional para gran parte de la población mundial debido a que proporciona alrededor del $20 \%$ de energía y el $15 \%$ de proteína percapita. Su producción y consumo lo han convertido en uno de los cultivos alimenticios más importante a nivel global (Martínez et al. 1997). Sin embargo, para responder a la demanda de este cereal, es necesario elevar los rendimientos en $70 \%$ por encima de los niveles actuales (Sanint 1998).

En Cuba, el incremento del rendimiento por área y el aumento de la producción hasta lograr el autoabastecimiento de arroz (500 mil toneladas de arroz consumo/

\footnotetext{
1 Recibido para publicación el 25 de noviembre del 2003.

2 Estación Territorial de Investigaciones del Arroz “ Jucarito”, Granma, Cuba.

3 Instituto de Investigaciones Agropecuarias “ Jorge Dimitrov”, Gaveta Postal 2140, Bayamo 85100, Granma, Cuba.
} 
año); es una meta de prioridad estatal para satisfacer el percápita anual de más de $45 \mathrm{~kg}$ (Suárez et al. 2000). En este sentido se cuenta con una tecnología de avanzada y se trabaja en la generación de variedades con características específicas para ser explotadas en los diferentes ecosistemas de producción (Avila et al. 1998 y Deus et al. 2000). Esto pudiera estar motivado a que, para los cubanos, el arroz representa un alimento básico en la dieta de la población, siendo el consumo percápita uno de los más elevados de América Latina, aún teniendo presente las regulaciones existentes (Gutiérrez 1998).

Para la obtención de variedades de arroz con mejores atributos que las existentes, se necesitan condiciones indispensables, tales como una estrategia adecuada de mejoramiento y una amplia diversidad genética para asegurar el rendimiento y la estabilidad del comportamiento, lo que se puede lograr por cruzamiento y posterior selección de los mejores genotipos (CIAT 1996, Diaz, Pérez y Morejon 1998).

En el presente trabajo se evaluó un grupo de variedades y/o líneas sobre la base de múltiples caracteres con el propósito de seleccionar las de mejor comportamiento, que puedan ser recomendadas para su explotación en áreas de producción de la zona oriental de Cuba.

\section{MATERIALES Y MÉTODOS}

La investigación se desarrolló con arroz irrigado en las áreas de la Estación Territorial de Investigaciones del Arroz (ETIA) "Jucarito", situada en el km 48 de la carretera Bayamo - Tunas, próximo a la comunidad de Vado del Yeso, municipio Río Cauto, provincia Granma. Esta zona se caracteriza por presentar dos períodos climáticos bien definidos; uno húmedo que se extiende desde mayo hasta octubre, en el que se produce cerca del $80 \%$ de las precipitaciones anuales y otro relativamente seco, que abarca los meses desde noviembre hasta abril, en el que apenas se rebasa el $20 \%$ de las lluvias anuales (Socorro 1989). La cantidad de precipitaciones para esta zona oscila entre 1.000 y $1.200 \mathrm{~mm}$ de agua; se alcanza una temperatura promedio anual de $26{ }^{\circ} \mathrm{C}$ y una evaporación media de $2000 \mathrm{~mm}$ (Pujol et al. 1990).

Los valores promedios acumulados, de las variables climáticas durante los meses de ejecución del experimento en cada etapa de estudio se muestran en el Cuadro 1.

En el trabajo se estudiaron 33 variedades: 15 de ciclo medio y 18 de ciclo corto, incluyendo las dos variedades utilizadas como testigo, J-104 para ciclo medio y la variedad Perla para ciclo corto.
La investigación se desarrolló en el campo, bajo un diseño de bloques completos al azar con cuatro réplicas. Se estudiaron simultáneamente por separado las variedades de ciclo medio y corto, durante las campañas de siembra seca 2001/2002 y húmeda 2002.

La siembra se realizó para la campaña seca en la primera quincena de enero y la de primavera en la primera quincena de julio de forma manual a chorrillo en surcos separados a $15 \mathrm{~cm}$ entre hilera, en parcelas de 3 x $5 \mathrm{~m}$, con una norma de siembra de $160 \mathrm{~kg} / \mathrm{ha}$ de semilla calculada al $100 \%$ de germinación, en un tipo de suelo Gley Nodular Ferruginoso Típico (Hernández 1995).

El manejo agrotécnico del cultivo (atenciones culturales, control de malezas, manejo del agua, control de plagas y enfermedades y cosecha) se realizó, según las recomendaciones técnicas establecidas por los Instructivos

Cuadro 1. Comportamiento de las variables climáticas durante el período de desarrollo del experimento. Cuba. 2001-2002.

\begin{tabular}{|c|c|c|c|c|c|c|}
\hline \multirow[t]{2}{*}{ Año } & \multirow[t]{2}{*}{ Meses } & \multicolumn{3}{|c|}{ Temperaturas } & \multirow{2}{*}{$\begin{array}{c}\text { Humedac } \\
\text { relativa } \\
\overline{\mathbf{x}}\end{array}$} & \multirow{2}{*}{$\begin{array}{l}\text { Precipitac. } \\
(\mathbf{m m})\end{array}$} \\
\hline & & Máxima & Mínima & $\overline{\mathbf{x}}$ & & \\
\hline \multirow{6}{*}{2} & Enero & 31,4 & 16,9 & 23,1 & 79,6 & 9,9 \\
\hline & Febrero & 30,7 & 17 & 22,7 & 76,0 & 16,8 \\
\hline & Marzo & 31,8 & 17,2 & 23,2 & 75,8 & 21,7 \\
\hline & Abril & 34,4 & 20,8 & 26,6 & 71,3 & 32,4 \\
\hline & Mayo & 34,2 & 21,6 & 24,3 & 71,6 & 234,5 \\
\hline & Junio & 32,9 & 18,4 & 26,9 & 79,5 & 216,4 \\
\hline \multirow[t]{2}{*}{0} & Julio & 32,8 & 20,6 & 25,9 & 81,5 & 202 \\
\hline & Agosto & 32,8 & 19,4 & 24,5 & 74,9 & 151,7 \\
\hline \multirow[t]{2}{*}{0} & Septiembre & 33,7 & 22,9 & 27,2 & 81,7 & 185,3 \\
\hline & Octubre & 33,8 & 23,0 & 27,7 & 85,7 & 35 \\
\hline \multirow[t]{6}{*}{1} & Noviembre & 31,7 & 20,2 & 25,0 & 82,6 & 33,7 \\
\hline & Diciembre & 31,7 & 19,3 & 24,5 & 85,4 & 1,5 \\
\hline & $\overline{\mathrm{x}}(\mathrm{año})$ & 32,7 & 19,8 & 25,1 & 78,8 & $1.140,9$ \\
\hline & Enero & 30,7 & 17,9 & 24,0 & 83,9 & 30,5 \\
\hline & Febrero & 30,2 & 16,5 & 22,9 & 77,5 & 55,5 \\
\hline & Marzo & 32,1 & 18,6 & 24,7 & 76,4 & 33,6 \\
\hline \multirow[t]{2}{*}{2} & Abril & 33,4 & 19,5 & 25,7 & 72,3 & 50,6 \\
\hline & Mayo & 34,5 & 22,4 & 27,2 & 79,3 & 96,5 \\
\hline \multirow[t]{2}{*}{0} & Junio & 33,8 & 19,5 & 27,0 & 77,5 & 193,1 \\
\hline & Julio & 35,1 & 23,4 & 28,4 & 79,6 & 152,5 \\
\hline \multirow[t]{2}{*}{0} & Agosto & 35,9 & 21,6 & 28,1 & 77,6 & 41,9 \\
\hline & Septiembre & 34,6 & 22,6 & 28,1 & 79 & 144,9 \\
\hline \multirow[t]{4}{*}{2} & Octubre & 32,9 & 22,4 & 28,1 & 84,4 & 53,2 \\
\hline & Noviembre & 30,4 & 20,6 & 27,1 & 84,5 & 44,1 \\
\hline & Diciembre & 29,8 & 16,2 & 24,9 & 83,7 & 7,1 \\
\hline & $\overline{\mathrm{x}}(\mathrm{año})$ & 32,8 & 20,1 & 22,9 & 79,6 & 903,5 \\
\hline \multicolumn{2}{|c|}{$\overline{\mathrm{x}}$ (período) } & 32,75 & 19,95 & 25,9 & 79,2 & $1.022,2$ \\
\hline
\end{tabular}


Técnicos para el arroz (Dirección Nacional de Arroz (1998). La cosecha se realizó cuando las variedades alcanzaron el $20 \%$ de humedad del grano en función del ciclo.

Después de cosechada la muestra de arroz se determinó en el laboratorio la humedad del grano, mediante el empleo del determinador de humedad "Steinlite", las muestras de arroz cosechadas para determinar el rendimiento agrícola, análisis industriales y la humedad del grano, fue cosechado por lo general en el horario de 9:00 a.m a las 12:00 a.m.

Se evaluó: el vigor vegetativo, tipo de planta, resistencia al acame, resistencia al desgrane, resistencia al insecto Tagosodes oryzicolus, resistencia al virus de la hoja blanca, resistencia a la enfermedad fungosa Pyricularia grisea, la altura de las plantas, la duración del ciclo y el rendimiento agrícola y sus componentes panículas por $\mathrm{m}^{2}$, granos por panícula y peso de 1000 granos, siguiendo la metodología del CIAT (1983). El rendimiento agrícola se determinó en un área de $8 \mathrm{~m}^{2}$ ( $2 \times 4 \mathrm{~m})$ en cada parcela, expresado en $\mathrm{t} / \mathrm{ha}$ al $14 \%$ de humedad del grano.El análisis industrial o de molinería para determinar los porcentajes de granos enteros y granos partidos se realizó mediante el procesamiento de un $\mathrm{kg}$ de arroz cáscara libre de impurezas, procedente de la muestra secada al sol.

Se realizaron análisis de varianza simple y pruebas de comparación múltiple de media por la prueba de Newman Kewll, usando el paquete estadístico STATISTIC.

La valoración económica se realizó, según la metodología usada por Peña (1998), sobre la base del rendimiento agroindustrial de las variedades por campaña experimental, teniendo en cuenta que el arroz entero se cotiza a $\$ 380.00$ la tonelada y el arroz partido a $\$$ 279.00 , en comparación con los valores de las variedades utilizadas como testigos.

\section{RESULTADOS Y DISCUSIÓN}

En el Cuadro 2 se presenta el rendimiento y sus componentes en las variedades de ciclo medio, para las dos campañas estudiadas. Con excepción del ciclo, se observaron diferencias significativas entre variedades para los restantes indicadores evaluados; lo que sugiere la existencia de variación genotípica; similar a lo señalado por diferentes autores en el cultivo del arroz (Pérez et al. 1989 a, 1989 b, Morales 1992).

El ciclo varió entre 134 - 141 para la campaña seca y entre 116 - 119 días para la campaña húmeda; va- lores que están en el rango señalado por Ismail y Deus (1990) en un estudio preliminar con 13 variedades y/o líneas de ciclo medio en la localidad de "Los Palacios. Estos autores al igual que en nuestro estudio, no encontraron diferencias significativas para el ciclo durante la época húmeda.

Con relación al rendimiento y el resto de los componentes las variedades IACuba-17 e IACuba-19 mostraron, junto con el testigo J-104, los mayores valores, sin diferencias significativas entre ellas y sí con respecto a las variedades IACuba-14 a IACuba-15 en las dos campañas estudiadas. Al respecto estudios realizados en diferentes localidades del país han indicado un potencial del rendimiento de estas variedades similar al de la variedad J-104; aspecto que puede ser explicado en cierta medida por la participación de J-104 como uno de los progenitores utilizados en su obtención.

La altura de las plantas varió entre 70-77 y entre $86-93 \mathrm{~cm}$ en las campañas seca y húmeda respectivamente, lo que es considerado normal para este tipo de material índico - semienano y característico, según Lajovskin et al. (1977), de las variedades tropicales.

En relación con las épocas merece destacar que siempre se observaron los mayores valores en el rendimiento y sus componentes en la época de seca, lo que coincide con otros autores cubanos como Ismail y Deus (1990), Morales et al. (1992), Avila et al. (1998), Deus et al. (2000) y Suárez et al. (2000). Tal comportamiento pudiera ser debido a que en la temporada seca hay una mayor duración del día, y al ser mayor la luminosidad, las plantas hacen una mayor utilización de la energía solar en los procesos fotosintéticos; lo que se traduce en mayores rendimientos.

La variedad IACuba-19, sobresalió del resto por presentar un vigor inicial excelente (Cuadro 3).

Frente a las principales plagas y enfermedades, todas las variedades mostraron mejor comportamiento que la J-104; aspecto este muy importante, pues la susceptibilidad que muestra la variedad J-104 frente al hongo Pyricularia grisea, sugiere su sustitución paulatina de las áreas de producción y las variedades IACuba-17 e IACuba-19, pudieran ser sus posibles sustitutos.

Con relación a la calidad del grano (Figura 1) las variedades IACuba-17 con $56 \%$ y IAcuba-19 con 55\% de granos enteros superaron estadísticamente a la variedad J-104, que logró 48\%, lo que de acuerdo con los lineamientos de la Dirección Nacional del cultivo es buena. Un comportamiento similar se observó con respecto a la cristalinidad del grano; donde el rango de las variedades estuvo entre 60-89\%, en relación con la J-104 
Cuadro 2. Comportamiento del rendimiento y sus componentes durante las campañas de seca 2001/2002 y la húmeda 2002, en las variedades de ciclo medio seleccionadas. Cuba, 2001-2002.

\begin{tabular}{|c|c|c|c|c|c|c|}
\hline Variedades $C$ & Ciclo (días) & $\mathbf{A P}(\mathbf{c m})$ & $\mathbf{P} / \mathbf{M}^{2}\left(\mathbf{N}^{\circ}\right)$ & Grll/P(No) & PMG (g) & $\mathbf{R A}(\mathbf{t} / \mathbf{h a})$ \\
\hline \multicolumn{7}{|c|}{ Campaña seca } \\
\hline IACuba 14 & $134 \mathrm{a}$ & $70.9 \mathrm{~b}$ & $413 \mathrm{~b}$ & $65,7 \mathrm{~b}$ & $29,0 \mathrm{~b}$ & $7,80 \mathrm{bc}$ \\
\hline IACuba 15 & $134 \mathrm{a}$ & $70,3 \mathrm{~b}$ & $413 \mathrm{~b}$ & $65,5 \mathrm{~b}$ & $29,2 \mathrm{~b}$ & $7,67 \mathrm{c}$ \\
\hline IACuba 17 & $141 \mathrm{a}$ & 76,7 a & 446 a & 68,5 a & $30,7 \mathrm{ab}$ & 9,40 a \\
\hline IACuba 19 & $138 \mathrm{a}$ & $75,7 \mathrm{a}$ & $447 \mathrm{a}$ & 66,5 a & $31,5 \mathrm{a}$ & 9,42 a \\
\hline J-104 (testigo) & $138 \mathrm{a}$ & 77,8 a & 441 a & $66,2 \mathrm{ab}$ & $31,5 \mathrm{a}$ & $8,40 \mathrm{~b}$ \\
\hline $\mathrm{DE}$ & $\pm 3,20 \mathrm{NS}$ & $\pm 3,90 * *$ & $\pm 27,11^{*}$ & $\pm 1,06^{* * *}$ & $\pm 0,53 * * *$ & $\pm 0,75^{* * *}$ \\
\hline \multicolumn{7}{|c|}{ Campaña húmeda } \\
\hline IACuba 14 & $117 \mathrm{a}$ & 86,9 b & $393 \mathrm{~b}$ & 53,5 a & $28,9 \mathrm{~b}$ & $5,10 \mathrm{c}$ \\
\hline IACuba 15 & $116 \mathrm{a}$ & 86,6 b & $391 \mathrm{~b}$ & 53,5 a & $29,0 \mathrm{ab}$ & $5,07 \mathrm{c}$ \\
\hline IACuba 17 & 118 a & 91,7 a & 412 a & $46,0 \mathrm{~b}$ & 30,1 a & 6,95 a \\
\hline IACuba 19 & 119 a & $90,6 \mathrm{ab}$ & $403 \mathrm{ab}$ & $49,2 \mathrm{ab}$ & 30,3 a & 6,82 aа \\
\hline J-104 (testigo) & ) $119 \mathrm{a}$ & 93,7 a & 424 a & $45,2 \mathrm{~b}$ & 30,5 a & $5,75 \mathrm{~b}$ \\
\hline $\mathrm{DE}$ & $\pm 1,88 \mathrm{NS}$ & $\pm 3,74^{* *}$ & $\pm 19,05^{*}$ & $\pm 4,35 * * *$ & $\pm 0,81^{* * *}$ & $\pm 0,18^{* * *}$ \\
\hline
\end{tabular}

* Para $\mathrm{P} \leq 0,05, * *$ Para $\mathrm{P} \leq 0,01, * * *$ Para $\mathrm{P} \leq 0,001$, Letras desiguales indican diferencias significativas por la prueba de Newman Kewls, $\mathrm{AP}=$ Altura promedio, ${ }^{\mathrm{NS}}=$ No significativo, $\mathrm{P} / \mathrm{M}^{2}=$ Panícula por metro cuadrado, Grll/P = Granos llenos por panícula, $\mathrm{PMG}=$ Peso de mil granos, RA = Rendimiento Agrícola .

que fue de $59 \%$. Las variedades IACuba-17 e IACuba19 se destacaron, además, por presentar los mayores valores de proteína en el grano (Figura 1B), en correspondencia con su comportamiento en diferentes localidades del país (Dirección Nacional de Arroz 1998).

En el Cuadro 4 se muestra el comportamiento de las variedades de ciclo corto seleccionadas. En común las variedades IACuba-18 e IACuba-20 no mostraron diferencias significativas en ninguno de los indicadores evaluados en la época de seca con la variedad Perla, usada como testigo, y en la campaña húmeda se observó sólo significación estadística para el ciclo y el peso de mil granos; donde las variedades fueron ligeramente superiores a la Perla.

Respecto al rendimiento agrícola, no hubo diferencias significativas entre variedades, pero se observó en ambas épocas una tendencia definida de las variedades IACuba-18 e IACuba-20 a presentar los mayores valores. Por otra parte los valores fueron siempre más altos en la campaña seca, con relación a la húmeda, en correspondencia con lo observado por Pérez et al. (1989) en un estudio de variedades de ciclo corto en la zona central del país y donde estuvo involucrada la variedad Perla. En este estudio las diferencias estacionales pudieron
Cuadro 3. Características agronómicas de las variedades y/o líneas de ciclo medio (campañas de frío y primavera). Cuba, 2001-2002.

\begin{tabular}{|c|c|c|c|c|c|c|}
\hline \multirow[b]{2}{*}{ Código } & \multirow[b]{2}{*}{ V. $V^{1}$} & \multirow[b]{2}{*}{ T.P } & \multicolumn{4}{|c|}{ Resistencia $^{2,3}$} \\
\hline & & & T.O & P.G & Ac & D \\
\hline 3001 & 3 & 3 & MR & MS & $\mathrm{R}$ & $\mathrm{R}$ \\
\hline 3292 & $3-2$ & 3 & MR & MS & $\mathrm{R}$ & $\mathrm{R}$ \\
\hline 3360 & 3 & 3 & MR & MS & $\mathrm{R}$ & $\mathrm{R}$ \\
\hline 3361 & 3 & 3 & MR & $\mathrm{S}$ & $\mathrm{R}$ & $\mathrm{R}$ \\
\hline 4322 & $3-4$ & 3 & MR & $\mathrm{S}$ & $\mathrm{R}$ & $\mathrm{R}$ \\
\hline 4342 & 3 & $4-2$ & MR & MS & $\mathrm{R}$ & $\mathrm{R}$ \\
\hline 4370 & $3-2$ & 3 & MR & MS & $\mathrm{R}$ & $\mathrm{R}$ \\
\hline 4372 & $3-2$ & 3 & MR & MS & $\mathrm{R}$ & $\mathrm{R}$ \\
\hline 4377 & 3 & 3 & MR & MS & $\mathrm{R}$ & $\mathrm{R}$ \\
\hline 4403 & 2 & 3 & MR & S & $\mathrm{R}$ & $\mathrm{R}$ \\
\hline $\mathrm{J}-104$ (testigo) & 3 & 3 & MR & MS & $\mathrm{R}$ & $\mathrm{R}$ \\
\hline IACuba 17 & 3 & 3 & $\mathrm{R}$ & MS & $\mathrm{R}$ & $\mathrm{R}$ \\
\hline IACuba 14 & 3 & 4 & $\mathrm{R}$ & MS & $\mathrm{R}$ & $\mathrm{R}$ \\
\hline IACuba 15 & 3 & 3 & $\mathrm{R}$ & MR & $\mathrm{R}$ & $\mathrm{R}$ \\
\hline IACuba 19 & $3-2$ & 3 & $\mathrm{R}$ & MR & $\mathrm{R}$ & $\mathrm{R}$ \\
\hline
\end{tabular}

${ }^{1} \mathrm{~V} . \mathrm{V}=$ Vigor vegetativo, $\mathrm{T} . \mathrm{P}=$ Tipo de planta, $\mathrm{T} . \mathrm{O}=$ Tagosodes ory zicolus, $\mathrm{P} . \mathrm{G}=$ Pyricularia grisea, Ac. Acame, $\mathrm{D}=$ Desgrane.

2 CIAT 1983.

${ }^{3} \mathrm{~S}=$ susceptible; $\mathrm{MS}=$ medianamente susceptible; $\mathrm{MR}=$ medianamente resistente; $\mathrm{R}=$ resistente. 

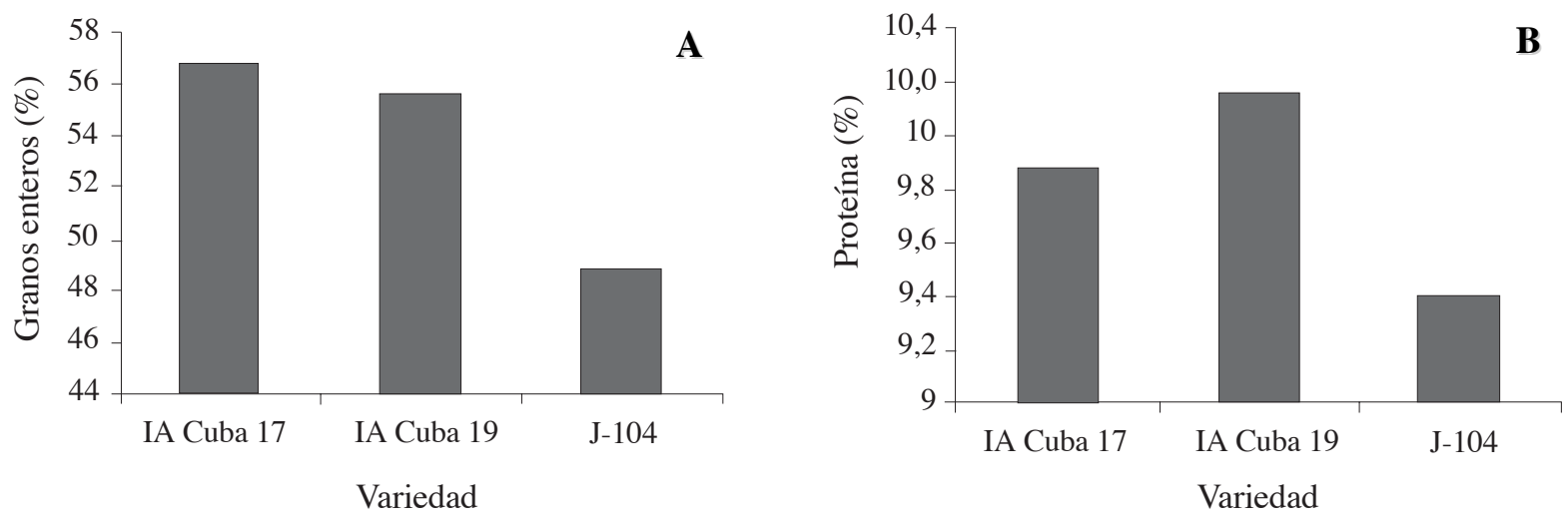

Figura 1. Porcentaje de granos enteros y proteína en las variedades de ciclo medio. Cuba, 2001-2002.

ser explicadas por la expresión del carácter rendimiento, que puede ser el resultado de las características genéticas del material y del efecto modificador de un ambiente dado. Al respecto, Orellana et al. (1987) indicaron que las condiciones ambientales influyen grandemente en el rendimiento agrícola y las variedades responden de forma diferente ante estas condiciones.

Respecto al vigor vegetativo (V.V) y al tipo de planta las variedades mostraron un comportamiento excelente (grados 1, 2, 3), sin diferencias significativas entre ellas, al igual que frente a las principales plagas y enfermedades que afectan al cultivo (Cuadro 5).

Con relación a la calidad del grano las variedades IACuba- 18 con $58 \%$ e IACuba- 20 con $59 \%$ fueron li- geramente superiores a la variedad Perla con 55\% en granos enteros y cristalinos; así como se destacaron por presentar un mayor porcentaje de proteína en el grano (Figura 2).

En estudios en diferentes localidades del país se ha demostrado que las variedades IACuba-18 e IACuba-20 presentan un potencial de rendimiento similar a la Perla, pero que a diferencia de esta última se destacan por poseer una alta estabilidad para producir granos enteros cuando son cosechadas fuera de los parámetros de cosecha con humedad del $14 \%$ (Dirección Nacional de Arroz 1998, Peña 1998 y Castillo et al. 2000); por lo que pueden ser utilizadas como nuevas variedades para las áreas de producción y así diversificar el fondo genético en explotación, todo lo cual contrarrestará los efectos

Cuadro 4. Comportamiento del rendimiento y sus componentes durante las campañas de seca 2001/2002 y la primavera 2002, en las variedades de ciclo corto seleccionadas. Cuba, 2001-2002.

\begin{tabular}{|c|c|c|c|c|c|c|}
\hline Variedades & Ciclo (días) & $\mathbf{A P}(\mathbf{c m})$ & $\mathbf{P} / \mathbf{M}^{2}\left(\mathbf{N}^{\circ}\right)$ & Grll/P(No) & PMG (g) & $\mathbf{R A}(\mathbf{t} / \mathbf{h a})$ \\
\hline \multicolumn{7}{|c|}{ Campaña seca } \\
\hline IACuba 18 & $123 \mathrm{a}$ & 67,5 a & 419 a & $63,2 \mathrm{a}$ & 28,8 a & $7,54 \mathrm{a}$ \\
\hline IACuba 20 & $127 \mathrm{a}$ & 67,5 a & $426 \mathrm{a}$ & 57,5 a & 29,0 a & 7,30 a \\
\hline Perla (testigo) & $125 \mathrm{a}$ & $68,7 \mathrm{a}$ & $405 \mathrm{a}$ & $62,5 \mathrm{a}$ & 28,8 a & $7,12 \mathrm{a}$ \\
\hline $\mathrm{DE}$ & $\pm 4,27 \mathrm{NS}$ & $\pm 1,98 \mathrm{NS}$ & $\pm 23,11 \mathrm{NS}$ & $\pm 4,20 \mathrm{NS}$ & $\pm 0,26 \mathrm{NS}$ & $\pm 0,36$ NS \\
\hline \multicolumn{7}{|c|}{ Campaña húmeda } \\
\hline IACuba 18 & $108 \mathrm{a}$ & 69,6 a & $342 \mathrm{a}$ & 54,2 a & $27,4 a b$ & $5,04 \mathrm{a}$ \\
\hline IACuba 20 & $108 \mathrm{a}$ & 69,8 a & $353 \mathrm{a}$ & $50,7 \mathrm{a}$ & $28,5 \mathrm{a}$ & 5,03 a \\
\hline Perla (testigo) & $105 \mathrm{~b}$ & 72,1 a & $361 \mathrm{a}$ & 49,0 a & $26,1 \quad b$ & $4,95 \mathrm{a}$ \\
\hline $\mathrm{DE}$ & $\pm 1,88^{* *}$ & $\pm 1,94 \mathrm{NS}$ & $\pm 36,06 \mathrm{NS}$ & $\pm 3,19 \mathrm{NS}$ & $\pm 0,61^{* *}$ & $\pm 0,16 \mathrm{NS}$ \\
\hline
\end{tabular}

* Para $\mathrm{P} \leq 0,05$, ** Para $\mathrm{P} \leq 0,01$, *** Para $\mathrm{P} \leq 0,001$. Letras desiguales indican diferencias significativas por la prueba de Newman Kewls. $\mathrm{AP}=$ Altura promedio, $\mathrm{NS}_{=}$No significativo, $\mathrm{P} / \mathrm{M}^{2}=$ Panícula por metro cuadrado, Grll/P = Granos llenos por panícula, $\mathrm{PAG}=$ Peso de mil granos, RA = Rendimiento Agrícola. 
Cuadro 5. Características agronómicas y de resistencia de las líneas de ciclo corto (campañas de frío y primavera). 2001-2002.

\begin{tabular}{|c|c|c|c|c|c|c|}
\hline \multirow[b]{2}{*}{ Código } & \multirow[b]{2}{*}{ V.V 1} & \multirow[b]{2}{*}{ T.P } & \multicolumn{4}{|c|}{ Resistencia $^{2,3}$} \\
\hline & & & T.O & P.G & Ac & D \\
\hline Perla (testigo) & 3 & 3 & MR & MR & $\mathrm{R}$ & $\mathrm{R}$ \\
\hline IACuba 18 & 3 & 3 & MR & MR & $\mathrm{R}$ & $\mathrm{R}$ \\
\hline IACuba 20 & 3 & 3 & MR & MR & $\mathrm{R}$ & $\mathrm{R}$ \\
\hline 30703 & 3 & MR & MR & $\mathrm{R}$ & $\mathrm{R}$ & \\
\hline 34973 & 3 & MR & MR & $\mathrm{R}$ & $\mathrm{R}$ & \\
\hline 35003 & 3 & MR & MR & $\mathrm{R}$ & $\mathrm{R}$ & \\
\hline 35013 & 3 & MR & MR & $\mathrm{R}$ & $\mathrm{R}$ & \\
\hline 35203 & 3 & MR & MR & $\mathrm{R}$ & $\mathrm{R}$ & \\
\hline 35233 & 3 & MR & MR & $\mathrm{R}$ & $\mathrm{R}$ & \\
\hline 35282 & 3 & MR & MR & $\mathrm{R}$ & $\mathrm{R}$ & \\
\hline 35433 & 3 & MR & MS & $\mathrm{R}$ & $\mathrm{R}$ & \\
\hline 35593 & 3 & $\mathrm{R}$ & MR & $\mathrm{R}$ & $\mathrm{R}$ & \\
\hline 36733 & 3 & $\mathrm{R}$ & $\mathrm{S}$ & $\mathrm{R}$ & $\mathrm{R}$ & \\
\hline 43103 & 3 & $\mathrm{R}$ & MR & $\mathrm{R}$ & $\mathrm{R}$ & \\
\hline 43123 & 3 & $\mathrm{R}$ & MR & $\mathrm{R}$ & $\mathrm{R}$ & \\
\hline 43963 & 3 & MR & MS & $\mathrm{R}$ & $\mathrm{R}$ & \\
\hline 44403 & 3 & MR & MS & $\mathrm{R}$ & $\mathrm{R}$ & \\
\hline 71953 & 3 & MR & MR & $\mathrm{R}$ & $\mathrm{R}$ & \\
\hline
\end{tabular}

${ }^{1} \mathrm{~V} . \mathrm{V}=$ Vigor vegetativo, $\mathrm{T} . \mathrm{P}=$ Tipo de planta, $\mathrm{T} . \mathrm{O}=$ Tagosodes ory zicolus, $\mathrm{P} . \mathrm{G}=$ Pyricularia grisea, Ac. Acame, $\mathrm{D}=$ Desgrane.

2 CIAT 1983.

${ }^{3} \mathrm{~S}=$ susceptible; $\mathrm{MS}=$ medianamente susceptible; $\mathrm{MR}=$ medianamente resistente; $\mathrm{R}=$ resistente.

adversos de la erosión genética que se presenta cuando a escala de producción se trabaja con pocas variedades.

La valoración económica fue realizada sobre la base del rendimiento agroindustrial de las variedades, teniendo en cuenta que el arroz entero se cotiza a razón de $\$ 380.00$ la tonelada y a $\$ 279.00$ el arroz partido. Del mismo se evidencia que la introducción de las variedades de ciclo medio IACuba-17 e IACuba-19 en áreas de producción permitirá obtener altos rendimientos económicos entre 800 - $903 \$$ /ha para la primera y entre $813-815$ \$/ha para la segunda (Cuadro 6). Este comportamiento se considera importante, dado que la variedad J-104, que ocupa la mayor área de siembra en la provincia Granma, además de la pobre calidad industrial ha mostrado una alta susceptibilidad al hongo $P y$ ricularia grisea.

Cuadro 6. Valor de la producción y rendimiento económico para las variedades de ciclo medio.2001-2002.

\begin{tabular}{llrrrc}
\hline Variedad & Época & \multicolumn{3}{c}{ Valor de la producción (\$) } & Rend. \\
\cline { 3 - 6 } & & $\begin{array}{c}\text { Granos } \\
\text { enteros }\end{array}$ & $\begin{array}{c}\text { Granos } \\
\text { partidos }\end{array}$ & $\begin{array}{r}\text { Total } \\
\text { económico } \\
\text { \$/ha }\end{array}$ \\
\hline \multirow{2}{*}{ J-104 } & Seca & $4.116,0$ & $1.476,0$ & $5.592,0$ & - \\
& Húmeda & $2.817,5$ & $1.000,5$ & $3.822,5$ & - \\
IACuba 17 & Seca & $5.264,0$ & $1.128,0$ & $6.382,0$ & 800,0 \\
& Húmeda & $3.892,0$ & 834,0 & $4.726,0$ & 903,5 \\
IACuba 19 & Seca & $5.275,2$ & $1.130,4$ & $6.405,4$ & 813,4 \\
& Húmeda & $3.819,2$ & 818,4 & $4.637,6$ & 815,6 \\
\hline
\end{tabular}

Con relación a las variedades de ciclo corto (Cuadro 7), los rendimientos económicos estuvieran entre $162-436$ \$/ha para la variedad IACuba-18 y entre 204 - 341 \$/ha para la IACuba-20, muy inferior a lo obtenido en las variedades de ciclo medio, lo que pudo estar motivado a que la variedad Perla de Cuba, usada como testigo se caracteriza por presentar un potencial de rendimiento agroindustrial elevado (Dirección Nacional de Arroz 1998); no obstante estas variedades pudieran
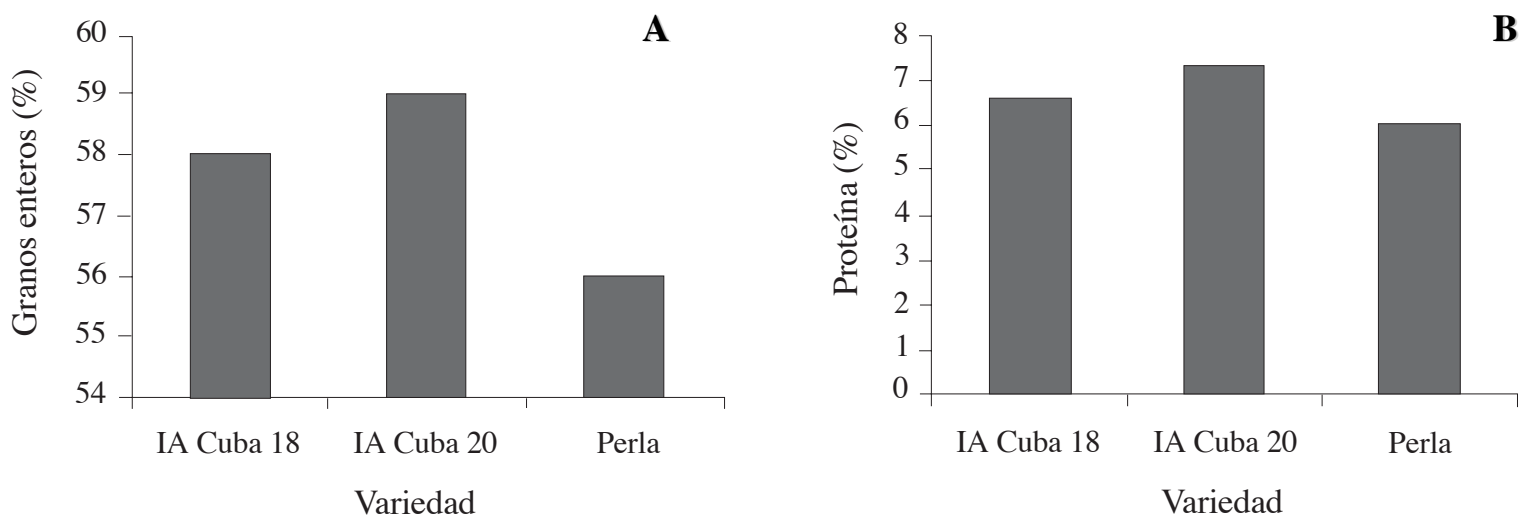

Figura 2. Porcentaje de granos enteros y proteína en las variedades de ciclo corto. 2001-2002. 
Cuadro 7. Valor de la producción y rendimiento económico para las variedades de ciclo corto.

\begin{tabular}{llcccc}
\hline Variedad & Época & \multicolumn{3}{c}{ Valor de la producción (\$) } & Rend. \\
\cline { 3 - 5 } & & $\begin{array}{c}\text { Granos } \\
\text { enteros }\end{array}$ & $\begin{array}{c}\text { Granos } \\
\text { partidos }\end{array}$ & Total & $\begin{array}{c}\text { económico } \\
\mathbf{\$} / \mathbf{h a}\end{array}$ \\
\hline \multirow{2}{*}{ Perla de } & Seca & $3.987,2$ & 854,4 & $4.841,4$ & - \\
Cuba & Húmeda & $2.772,0$ & 594,0 & $3.366,0$ & - \\
IACuba 18 & Seca & $4.373,2$ & 904,8 & $5.278,0$ & 436,6 \\
& Húmeda & $3.923,2$ & 604,8 & $3.528,0$ & 162,0 \\
\multirow{2}{*}{ IACuba 20 } & Seca & $4.307,0$ & 876,0 & $5.183,0$ & 341,6 \\
& Húmeda & $2.967,0$ & 603,6 & $3.570,6$ & 204,6 \\
\hline
\end{tabular}

diversificar la variabilidad genética y contrarrestar la vulnerabilidad a las enfermedades que se produce cuando existe una alta uniformidad genética entre las variedades que se explotan en condiciones de producción.

En su conjunto, los resultados agronómicos y económicos señalan la posibilidad de recomendar las variedades de ciclo corto IACuba-18 e IACuba- 20; y las variedades de ciclo medio IACuba-17 e IACuba-19 para ser explotadas en condiciones de producción en la provincia Granma.

\section{CONCLUSIONES}

Las variedades de ciclo corto IACuba-18 e IACuba-20, resultaron ser similar en rendimiento agrícola y resistencia a las principales plagas y enfermedades a la variedad Perla usada como testigo, pero superiores en granos enteros y cristalinos, así como en el porcentaje de proteínas en el grano. Esto, unido a la alta estabilidad que han presentado en la producción de granos enteros cuando son cosechadas fuera de los parámetros de cosecha, sugieren su utilidad como nuevas variedades para las áreas de producción y así diversificar el fondo genético en explotación.

Entre las variedades de ciclo medio se destacaron las variedades IACuba-17 e IACuba-19, que aunque no mostraron diferencias significativas en el rendimiento agrícola con la variedad testigo J-104, sí la superaron en el rendimiento industrial y la resistencia a enfermedades; aspectos muy importantes, pues la susceptibilidad que muestra la variedad J-104 frente al hongo Pyricularia grisea, sugiere su sustitución paulatina de las áreas de producción y estas variedades pudieran ser sus posibles sustitutas.

La valoración económica sobre la base del rendimiento agroindustrial permitió constatar la posibilidad de obtener rendimientos económicos superiores a 800 $\$ /$ ha con las variedades de ciclo medio IACuba-17 e IACuba-19 y superiores a $162 \$ /$ ha con las variedades de ciclo corto IACuba -18 e IACuba-20.

\section{LITERATURA CITADA}

AVILA, J.; PEÑA, L. R.; OBIOL, T.; VALERA, J.; SUÁREZ, I. 1998. Resultados del mejoramiento genético del arroz en la región más oriental de Cuba en el período 1993-1997. In: Memorias del I Encuentro Internacional de Arroz, La Habana. Cuba. pp. 46-52.

CIAT.1983. Sistema de evaluación estándar para arroz, Cali, Colombia.Centro de Investigaciones en Agricultura Tropical (CIAT). 140 pp.

CASTILLO, D.; HERNÁNDEZ, A.; BENEDITO DE BARBER, C.; BARBER, S. B.; MARTÍNEZ, J.; DUFFAY, I. 2000. Criterios sobre la calidad del arroz en Cuba. Revista Cubana del Arroz 2: 13-18.

CIAT. 1996. Improved rice germoplasm Latin America and the Caribbean. Annual Report. Centro de Investigaciones en Agricultura Tropical (CIAT). $86 \mathrm{pp}$

DEUS, J. E.; SUÁREZ, E.; ALFONSO, R.; HERNÁNDEZ, J.; PULDÓN, V. 2000. Algunas consideraciones sobre las variedades que conforman la estructura varietal actual. Revista Cubana del Arroz 1: 30-36.

DÍAZ, S., N. PÉREZ Y R. MOREJON. 1998. Evaluación de líneas de arroz (Oryza sativa L.) procedentes de los estudios superiores de rendimiento. Cultivos Tropicales 19(3): 61-63.

DIRECCIÓN NACIONAL DE ARROZ. 1998. Instructivo técnico para el cultivo del arroz. La Habana. Cuba. 58 pp.

GUTIÉRREZ, A. 1998. Palabras pronunciadas en la apertura del I Encuentro Internacional de Arroz. In: Memorias del I Encuentro Internacional de Arroz, La Habana. Instituto de Investigaciones del Arroz. pp. 7-10.

HERNÁNDEZ, A. 1995. Nueva versión de la clasificación de suelo. Ministerio de la Agricultura, 42pp.

ISMAIL, C.; DEUS, J. E. 1990. Estudio preliminar de líneas y variedades de arroz de ciclo medio en la localidad de "Los Palacios". Ciencia y Téc. Agric. Arroz 13(1-2): 85-93.

LAJOVSKIN, V.; LEYVA, B.; DEUS, J. 1977. Genofondo del arroz en Cuba. In: Memorias del I Taller Nacional en el cultivo del arroz. La Habana. IIA. pp. 23-26.

MARTÍNEZ, P.; TOHME, J.; LÓPEZ, J.; BORRERO, J.; MCCOUCH, S.R.; ROCA, W.; CHATEL, M.; GUIMARÃES, E. 1997. Estado actual del mejoramiento del arroz mediante la utilización de especies silvestres 
de arroz CIAT. In: Memorias de la Conferencia Internacional de Arroz para América Latina y El Caribe. Colombia, Bogotá. pp. 22-26.

MORALES, U.; MORENO, I.; PINO, M. 1992. Uso del coeficiente de sendero y del análisis de varianza en la caracterización y comparación de variedades de arroz en condiciones óptimas de cultivo. Cultivos Tropicales 13(1): 52-56.

ORELLANA, P.; ISJACOV, T; PÉREZ, J.; PÉREZ, R.; PRIETO, J.; SUÁREZ, E.; ISMAIL, C. 1987. Reacción de variedades de arroz (Oryza sativa L.) a diferentes condiciones ambientales. Centro Agrícola, 14(3): 15-20.

PÉREZ, R.; REYES, H.; HERNÁNDEZ, F. 1989 a. Comportamiento de variedades de arroz de ciclo corto en condiciones de riego. Centro agrícola 16(3): 55-63.

PÉREZ, R.; REYES, H.; RODRÍGUEZ, M. 1989 b. Comportamiento de seis variedades de arroz en condiciones de secano en Sancti Spíritus. Centro Agrícola 16(2):94-95.

PEÑA, L.R. 1998. Madurez óptima para la cosecha de las variedades de arroz IACuba 20 e IACuba 17 y su influen- cia sobre el rendimiento agrícola e industrial y el porcentaje de granos enteros en el proceso de molinería. Tesis en opción al título de Máster en Producción Vegetal. Universidad de Granma, Facultad de Ciencias Agrícolas. $86 \mathrm{p}$.

PUJOL, L; PAVÓN, A.; HERRERA, J.; ESPINOSA, E.; PÉREZ, A. 1990. Efectividad del aniego en el comportamiento de las aguas fréaticas en el Lote Piloto de Vado del yeso. Ciencia y Técnica en la Agricultura. Riego y drenaje 13(2):71-78.

SANINT, L. R. 1998. La economía del arroz en América Latina hacia el siglo XXI. In: Memorias del I Encuentro Internacional de Arroz, La Habana. Cuba. pp. 3-7.

SOCORRO, M. 1989. Características edafoclimáticas de las zonas arroceras de Cuba. Agrotécnica de Cuba 20(2): 37-61.

SUÁREZ, E; DEUS, J.E; PÉREZ, R.; AVILA, J.; HERNÁNDEZ, J.; ALFONSO, R.; PÉREZ, A.; PULDÓN, V. 2000. Resultados del Programa de Mejoramiento Genético del Arroz en Cuba durante el período 93-97. Revista Cubana del Arroz 1: 24-30. 